\title{
Graines souterraines ? Le sexo-générique dans une œuvre (auto)-censurée d'Anne Hébert : Les Songes en équilibre
}

Neil B. Bishop, Memorial University of Newfoundland / APFUCC 


\section{Pourquoi « Graines souterraines »?}

- Métaphore « saskatchewanaise » (colloque APFUCC à Saskatoon en 2007) qui énonce nos questions de base :

- 1. Quelles sont les configurations sexogénériques dans notre corpus?

- 2. Y a-t-il dans ce corpus des configurations sexo-génériques qui se retrouveront ailleurs dans l'oeuvre hébertienne - et le cas échéant : lesquelles? 


\section{Pourquoi « Power Point »?}

\section{Rendre le présent travail plus largement accessible.}




\section{Contexte}

- Actuellement : Intérêt renouvelé pour le sexo-générique dans l'œuvre d'Anne Hébert (Cahiers Anne-Hebert : numéro 8 consacré à ce thème)

- Il existe une longue tradition : lectures féministes de l'oeuvre

- Mises en question de ces lectures féministes

1. contestation du féminisme des Fous de Bassan (Marilyn Randall) ;

2. mise en relief des personnages masculins (Julie Gingras)

Contexte de Transdisciplinarité : visite à MUN (janvier 2007) de Christine OVERALL, philosophe féministe (Queen's, N-B) 


\section{La question précise : Vers un au-delà du sexo- générique ?}

Source conceptuelle : deux travaux de Christine OVERALL : "Return to Gender: Address Unknown » et «Gendering the University »

1. "gender » (ici « genre ") = construction sociale ( "On ne naît pas femme, on le devient » beauvoirien :

1.1 genre = ensemble de comportements professionnels, familiaux, vestimentaires et autres rattaché, voire imposé par une société aux personnes selon leur sexe biolegique

1.2 Overall : «Gender - S » : «le genre-social », le genre tel que défini ci-dessus ; ici : sigle «GS »

1.3 Overall: «Gender - I » : le «Genre - Je » auquel un individu se sent appartenir ou choisit d'appartenir - soit un des deux genres sociaux traditionnels, un mélange des deux ou (théoriquement) quelque chose de totalement différent (ici : GJe) 


\section{Rajouts aux concepts d'Overall : notions et sigles}

1. $\quad \mathrm{RGT}=$ "rôles génériques traditionnels " qui comportent $(\mathrm{OF}) \mathrm{H}$ (« oppression des femmes par les hommes) ;

2. RGNT (« rôles génériques non-traditionnels » qui comporteraient le dépassement du genre et l'épanouissement dans l'uchronie de l'au-delà du genre (DG = dépassement du genre social)

3. Sx = présence des deux sexes dang un texte

(rappel : RGT \{ par définition GS et (OF)H qui, eux, feront l'objet d'une mention spécifique ici quand leur mise en relief dans un texte rendra utile cette redondance [ = souvent]).

4. Configurations possibles (posons que le symbole $\{=$ « comprend», « comporte $», \ll$ inclut $»$; 


\section{Configurations possibles (suite)}

1. Formulations de base viturellement possibles

- $\mathrm{T}\{\mathrm{Sx}+\mathrm{RGT}$; $\mathrm{T}\{\mathrm{Sx}+\mathrm{RGT}+\mathrm{RGNT}$;

- $\quad$ T $\{$ Sx + RGNT

2. Nos Formulations emphatiques pour exprimer une intensité particulière d'une composant $\epsilon$ ( hahérente aux configurations de base,

- $\quad \mathrm{T}\{\mathrm{Sx}+\mathrm{RGT}+(\mathrm{OF}) \mathrm{H} ; \mathrm{T}\{\mathrm{Sx}+\mathrm{RGT}+\mathrm{GS}$

- $\quad \mathrm{T}\{\mathrm{S} x+\mathrm{RGT}+\mathrm{GS}+(\mathrm{OF}) \mathrm{H}$

- $\quad$ Configuration mixte T $\{\mathrm{Sx}+\mathrm{RGT}+(\mathrm{OF}) \mathrm{H}+\mathrm{RGNT}+\mathrm{DG}$

- $\quad T\{S x+R G N T+D G+G J e$ 


\section{Corpus : paramètres, limites}

- 1. présence de la problématique sexo-générique définie ci-dessus

- 2. " censure " refus chez Hébert de laisser republier certains textes qu'elle a fait paraître

3. vu limites de temps ; + AH fut d'abord reconnue pour le recueil de poèmes Les Songes en équilibre (publié et couronné du prix David en 1942) ; = ouvrage dont elle n'a jamais souhaité ni permis la re-publication : ce recueil sera notre corpus. 


\section{Les songes en équilibre : quatre parties («Songes », «Enfants », « Prières », "L'oiseau du poète ».}

- Problématique sexo-générique : dès le $2 e$ poème, «Les deux mains »; nous mettons en bleu les titres et citations hébertiens :

Cette main d'enfant, Cette main de femme.

Et parfois cette main travailleuse

Simple comme une main d'homme

Donc : présence des deux sexes (Sx); rapprochement femme-enfant (que seul sépare un signe de ponctuation faible : virgule); mais séparation marquée femme-homme (ponctuation forte : point).

S’opposent : entité [femme+enfant] ; entité [« main travailleuse » masculine].

Cette opposition reflète une répartition traditionnelle des rôles génériques, donc RGT. 


\section{« Les deux mains » (suite)}

MAIS le sujet locuteur (désormais SL) est en proie à une crise d'identité qu'exprime la métaphore d'une surabondance de «mains ».

SL « [...] découvre un nombre infini / En moi / De mains qui se tendent / Vers moi, / Comme des étrangères / Dont on a peur ».

Ces vers évoquent sans doute la perplexité ressentie par une jeune personne qui doit choisir son chemin dans la vie, $y$ compris son chemin générique. SL rêve de se débarrasser de

" toutes mes mains inutiles ", mais surtout de retrouver "Mes deux mains unies [;] Et le rivage / Qu'on touche Des deux mains, / Dans le même appareillage. 》 
«Les deux mains » (suite 2)

- "Les deux mains " thématise une quête actantielle : l'objet de valeur du Sujet désirant serait d'échapper à l'espace-temps de son présent et aussi,

- de dépasser la dichotomie générique pour aborder sur la "rive » accueillante de l'uchronie / l'utopie de l'au-delà du genre.

- SL exprime son espoir d'y trouver l'harmonie, un bonheur grâce au dépassement des tensions inhérentes à la dichotomie des $\underline{\mathrm{RGT}}$ 
- Le poème «Espace 》 présente le sexe masculin en rapport étroit avec SL :

- «Mes frères les Morts, / Donnez-moi la main, / Pour avancer sur les eaux, / Dans cet espace étendu entre les sphères ", espace sis « en moi » et qui se transmue par métaphore, en " pleine mer », " Au milieu de l'Océan » - sans « rive » ni « rivage » (donc, chronotope récurrent)

- L'espace éponyme est dysphorique : SL est perdu dans un univers menaçant, sans autres adjuvants que des " morts" dans sa quête sans objet de valeur précis : quête confuse, tant la perte de tout repère est absolue.

- «Espace » textualise : crise spirituelle autant qu'identitaire, et, ainsi, sape l'adhésion aux certitudes catholiques que le recueil exprime ailleurs. Par là, " Espace » conteste le règne des RGT. 
«Mort » évoque le passage dysphorique d'une pensée magique enfantine à la pensée maqique chrétienne.

- SL est féminin : («Mes fées / M’ont quittée / Et je suis restée seule / Avec un grand Christ / Entre les bras.» (nous soulignons)

- Cette voix féminine déplore sa perte d'un « réseau de soutien » féminin composé de « fées".

- Suite à cette perte, les longs cheveux du SL « collent » à la " plaie » du personnage christique (donc, masculin).

- Ses «larmes " ne sauraient libérer ses cheveux du sang et du cœur où, dit-elle, « Je me fige ».

- Cette situation dysphorique, où la femme est privée de liberté et de pouvoir, est conforme aux rapports de pouvoir inhérents aux RGT,

- $\mathrm{T}\{\mathrm{Sx}+\mathrm{RGT}+(\mathrm{OF}) \mathrm{H}$, bien plus nettement que dans les autres poèmes étudiés jusqu'ici - la mise en relief “ $(\mathrm{OF}) \mathrm{H}$ » s'impose.

- Mais ce poème sape aussi l'adhésion catholique que ce receuil exprime ailleurs, et donc conteste à son tour les RGT puisque RGT et catholicisme allaient de pair à l'époque. 
La deuxième section du recueil, "Enfants ", s'ouvre par "Oh! Mes joies enfantines! " qui, comme " Mort ", thématise la sortie d'une enfance euphorique + l'opposition entre les sexes.

- Les "joies enfantines ", personnifiées et féminisées comme de " [p]etites nymphes" (sœurs des "fées " du poème précédent), sont "disparues " sous l'eau, laissant " des ronds " à la surface.

- SL ajoute : "Un vieux professeur / Tout sec, tout gris, / Ajuste ses lunettes / [...] II regarde [...] ces cercles qui s'effacent, / Et m'explique que ce doit être / De la géométrie... ".

- Un verbe cognitif -- «expliquer » signale que ce n'est plus la pensée magique chrétienne qui dispute la place à la pensée magique païenne - mais la pensée scolaire scientifique.

- SL s'affirme en tant que sujet féminin qui regimbe contre le monopole, par les hommes, du statut de maître à penser

- il conteste ce statut par sa présentation péjorative du professeur. 


\section{Apogée des RGT : « Maman » et «Berceuse lente »}

- Les quelques vers de «Berceuse lente » ci-dessous sont non seulement affirmatifs

- mais impératifs :

- en se présentant comme énonçant l'ordre du monde, ils intiment au lectorat l'ordre de se plier au rẻgne du Genre Social qui, chez chacune et chacun, doit devenir son GJe : la liberté individuelle est interdite au profit du GS :

- «Ton père retourne la terre, Et retourne les gerbes, / II a des peines et des soucis. / Pour que tout soit tranquille / Les mamans / Bercent les enfants / Et les peines de l'époux 》

- L'homme est donc celui qui travaille à l'extérieur et qui a des fardeaux physiques et mentaux ; la femme est celle qui s'occupe - sans " soucis» ni «peines », apparemment -- des enfants et de la maison, et qui fournit le « repos du guerrier». Donc : puissante domination de GS.

- $\mathrm{T}\{\mathrm{Sx}+\mathrm{RGT}+\mathrm{GS}$ ou, pour simplifier, $\mathrm{T}\{\mathrm{Sx}+\mathrm{RGT}(\mathrm{GS})$ 
"Prières » : l'analyse de cette troisième section du recueil se heurte à son tour à la problématique de la place et des fonctions du catholicisme :

- Sans porter la charge de christianophobie de Kamouraska, des Enfants du Sabbat et des Fous de Bassan (entre autres), " Prières " n'exprime toutefois pas que l'adoration.

- Dans «Prière " (1er poème de la section) SL exprime le souci de son identité personnelle de Sujet au lieu d'en rester au thème chrétien traditionnel du « je ne suis rien, Vous Dieu êtes tout »:

- "En moi / Je ne sais plus où je suis, / Je ne sais plus où vous finissez, / Je ne sais plus si j'existe»

- Cette perplexité implique un désir de devenir / redevenir Sujet et aussi une mise en question des dogmes chrétiens traditionnels. Ici,

- $\mathrm{T}\{\mathrm{Sx}+\mathrm{RGT}+(\mathrm{OF}) \mathrm{H}$, mais la Femme interroge les limites de son GS définies par le catholicisme et se cherche un GJe de Sujet auto-défini. 


\section{«Sainte Vierge Marie » : poème révolutionnaire?}

- II valorise Marie, mère de Jésus, mère dont le dévouement à son fils était d'autant plus admirable qu'elle était pauvre. Par là,

- $T\{S x+R G T$

- «Révolutionnaires » (pour le Québec de l'époque) : deux énoncés.

- L'un transforme la Sainte-Trinité en une Sainte-Tétralogie, car il intègre Marie dans l'ensemble formé par les trois autres personnes divines. Marie doit cette promotion à son sexe féminin, le seul à pouvoir donner naissance non seulement à Dieu, mais aussi au sacrement fondamental de l'Église :

- «Surabondance du don parfait de Marie, / Et du Père et du Fils, / Et du Saint-Esprit, / Nous avons l'Eucharistie. »

- Promotion hébertienne de Marie, qui devient ici l'égale de l'ensemble Père + Fils + Saint-Esprit, l'égale donc de Dieu. 


\section{Deuxième promotion : l'accession de la femme au statut de prêtre [= contestation de RGT +GS]}

- Le deuxième énoncé « révolutionnaire »:

- "Quand je communie, / II me semble que c'est Marie / Qui me tend l'hostie. "

- Dans les deux cas, Hébert effectue une promotion de la femme, en lui permettant d'occuper un RGT réservé aux hommes, ce qui sape un RGT fondamental des sociétés catholiques.

- Dans le deuxième cas, il s'agit même d'une éviction (fantasmé) de I'homme par la femme : (OH)F. S'affirme ainsi la formule

- $\quad \mathrm{T}\{\mathrm{Sx}+\mathrm{RGNT}(\mathrm{DG})+(\mathrm{OH}) \mathrm{F}$.

- L'oeuvre d'AH comporte fort souvent (OF)H, mais parfois (OH)F. On observe donc ici deuX " graines souterraines » qui germent dans ce poème et qui pousseront ailleurs dans l'oeuvre. 


\section{4ième et dernière partie de $S E$ : « L'oiseau du poète »}

- Des « gens » vilipendent le poète éponyme :

- «Cependant les gens [...] s'agitent / Et jettent des pierres [...] / 'Que fait cet homme agenouillé, immobile, / Quand l'ennemi est aux portes de la ville? / C'est des soldats qu'il nous faut, / Quoi, c'est ça, l'homme ? [...] Qu'il reste où il est ! [...] Qu'il reste là / Avec les femmes, / Puisqu'il est inutile !' ”

Le SL cite un SL2 implicitement masculin puisque SL2 établit une séparation entre lui et «les femmes »: "Qu’il reste là ».

- La dévalorisation du poète accompagne la dévalorisation des femmes et des enfants en vertu de leur commune inutilité ! 
Ce « poète » manifeste ainsi un bouleversement générique socialement

imposé. SL valorise la dévirilisation du poète et sa transgénérisation:

- "On le méprise. Et pourtant que ferait-on / Sans lui ? / Qui garderait le feu / Sans la Vestale au temple ? / II accepte l'injure. I Pour son pays il sera / L'offrande recueillie / D'une âme qui prie. 》

- « accepter l'injure » = faire le sacrifice de son statut générique masculin au profit du statut de femme ("Vestale ») c-à-d d'un RGT dévalorisé par le sexe dominant de la société fictive du poème. Il y a du Genre-Je chez ce poète, puisqu'il " accepte » et se constitue en " offrande ", acte de sujet.

- À la fin, ce poète féminisé devient, grâce à un don de Dieu (à savoir "De quoi faire un poème") un " poète en état de grâce " et passe à la création. Ce don de création $\simeq$ la fonction maternelle). 
«L'oiseau du poète » = configuration bivalente

T $\{$ Sx + RGT(OF)H + RGNT(DG)

- SL valorise ce RGNT + DG du poète qui passe - de force ET de gré -- de

- $\mathrm{GS}=\mathrm{H}$ à GS et GJe = F. 
Corpus présente une prédominance massive de T \{ Sx + RGT (GS) ; GS est souvent si oppressif de la femme qu'il faut ajouter (OF)H pour le souligner: $T\{S x+R G T(G S)(O F) H$

- Une différence importante entre Les Songes en équilibre et une large part du reste de l'œuvre : dans de nombreux textes hébertiens ultérieurs, la révolte contre RGT + GS + (OF)H sera à l'avant-plan thématique. Cette évolution, et l'éloignement de la pensée de l'auteure par rapport au catholicisme, expliquent en partie, sans doute, la censure dont Anne Hébert a frappe ce recueil, quoique dans une entrevue avec Robidoux et Major (1976), elle ait plutôt évoqué des considérations formelles.

- Toutefois, enfouis déjà dans Les Songes en équilibre -- qui constitue la nuit des temps, le souterrain de l'œuvre hébertienne gisent, vivaces, les graines de la révolte, graines de RGNT (DG).

- Graines de RGT et de RGNT + DG + GJe s'écloront pour donner une richissime moisson littéraire dynamisée par la lutte entre l'actuel référentiel de RGT et l'uchronie d'un monde de RGNT, de DG et de GJe à faire advenir. 


\section{Bibliographie}

- $\quad$ BEAUCHEMIN, Mélanie. 2007. Dynamiques transgressives et manifestations de la femme maléfique dans l'oeuvre d'Anne Hébert, thèse, Université de Sherbrooke.

- $\quad$ BISHOP, Neil B. 1993. Anne hébert, son œuvre, leurs exils, Talence, Presses Universitaires de Bordeaux.

- $\quad$ GINGRAS, Julie 1997 Situation du personnage masculin dans les romans d'Anne Hébert, mémoire de M.A., Montréal, Université McGill.

- HARVEY, Robert. 2000. Poétique d'Anne Hébert. [...]. Québec, l’Instant même, pp. 29-60.

- $\quad$ HÉBERT, Anne. 1942. Les Songes en équilibre, Montréal, Éditions de l'arbre. , 1976, Entrevue audio-visuelle avec Jean-Louis Major et Réjean Robidoux, University of Toronto.

- OFFICE QUÉBÉCOIS DE LA LANGUE FRANÇAISE. 2007. Dictionnaire de dépannage linguistique, http://66.46.185.79/bdl/gabarit bdl.asp?t1=1\&id=2387\&D=Genre.

- $\quad$ OVERALL, Christine 2000 « Return to Gender, Address Unknown : Reflections on the Past, Present, and Future of the Concept of Gender in Feminist Theory and Practice", in Estes, Y., A. L. Farr, P. Smith et C. Smyth (dir.), Marginal Groups and Mainstream American Culture, Lawrence, University Press of Kansas.

- $\quad$ 2007. "Sexism and the Gendering of the University », conférence prononcée à la Memorial University of Newfoundland le 19 janvier 2007.

- RANDALL, Marilyn. 1989 «Les énigmes des Fous de Bassan : féminisme, narration et clôture », Voix et images, 43 : 66-82.

- $\quad$ RUSSELL, Delbert W. 1983. Anne Hébert, Boston, Twayne Publishers. 